EPJ Web of Conferences 41, 07013 (2013)

DOI: $10.1051 /$ epjconf/20134107013

(C) Owned by the authors, published by EDP Sciences, 2013

\title{
Measuring enzyme binding using shaped ultrafast laser pulses
}

\author{
B. J. Pearson ${ }^{1}$, C.-H. Tseng ${ }^{2}$, and T. C. Weinacht ${ }^{2}$ \\ ${ }^{1}$ Department of Physics and Astronomy, Dickinson College, Carlisle, PA, 17013, USA \\ ${ }^{2}$ Department of Physics and Astronomy, Stony Brook University, Stony Brook, NY, 11794, USA
}

\begin{abstract}
We use multiphoton quantum-control spectroscopy to discriminate between enzyme-bound and unbound NADH (reduced nicotinamide adenine dinucleotide) molecules in solution. Shaped ultrafast laser pulses are used to illuminate both forms of $\mathrm{NADH}$, and the ratio of the fluorescence from the bound and unbound molecules for different pulse shapes allows us to measure binding without spectrally resolving the emitted fluorescence or relying on the absolute fluorescence yield. This permits determination of enzyme binding in situations where spectrally resolved measurements and absolute fluorescence yields are difficult to obtain, and makes the approach ideal for multiphoton microscopy with molecular discrimination.
\end{abstract}

\section{Introduction}

In chemical or biological applications, quantum control using shaped ultrafast laser pulses can be thought of as a path toward discrimination, where the shaped pulses selectively distinguish between different sub-ensembles of a population [1]. The general technique is known as quantum-control spectroscopy [2], where a molecular response is measured as a function of pulse shape instead of the frequency of a single excitation source. In this paper we use quantum-control spectroscopy to discriminate between unbound and enzyme-bound forms of the metabolically important, intrinsically fluorescent biomolecule NADH (reduced nicotinamide adenine dinucleotide) [3]. By exciting the sample with two pulse shapes in succession, we are able to rapidly determine enzyme binding of NADH without needing to measure the fluorescence spectrum or absolute yield. As discussed in more detail below, the approach is ideal for multiphoton microscopy with molecular discrimination and should be generally applicable to any fluorescent biological or chemical molecule that can be two- or three-photon excited by near-infrared light.

\section{Experimental approach}

Laser pulses from an amplified, ultrafast laser system are shaped in an acousto-optic pulse shaper, split with a beam splitter, and directed into cuvettes that contain either NADH alone in Trizma buffer or NADH plus the enzyme MDH (malate dehydrogenase) in $3.2 \mathrm{M}$ ammonium sulphate solution and Trizma. The two-photon fluorescence at 90 degrees is collected by photomultiplier tubes and recorded by a computer. Our goal is to discriminate between the unrestricted use, distribution, and reproduction in any medium, provided the original work is properly cited. 
unbound and enzyme-bound forms of $\mathrm{NADH}$, and despite the fact that the two-photon absorption spectra are essentially the same for the two forms [4], we find that subtle differences in their responses to a shaped pulse allow us to easily distinguish between them [5]. The specific pulse shape we use is a spectral phase step of $\pi$ radians at a given location in the excitation spectrum, an approach motivated by applications in atomic and molecular physics [6].

\section{Results and discussion}

Figure 1 (left panel) shows the normalized fluorescence as a function of $\pi$-phase step position across the laser bandwidth for both the unbound and enzyme-bound molecular solutions. We find that the control exploits the "double-minima" structure in the curves, as the relative depth between the two minima depend on the binding. This can be seen more clearly in the right panel of Fig. 1, which plots the ratio of the signals at the two minima in the curves as the enzyme concentration is increased. An estimate of the binding fraction is shown across the top of the graph [7]. The net result is that the short-wavelength minimum becomes less pronounced as the enzyme concentration increases. This monotonic behaviour allows one to exploit the fluorescence ratio from two different pulse shapes to determine the extent of enzyme binding in the laser focus.

Specifically, our results show promise for microscopy experiments where changes in molecular function or transformation (e.g. enzyme binding) need to be monitored in real time. Rapidly switching between two (or more) pulse shapes with software modifications allows one to discriminate between molecular subspecies on a near shot-by-shot basis. Although the degree of control in a given trial may be modest, the experiment readily achieves the essential feature of control exceeding the noise that is required for discrimination.
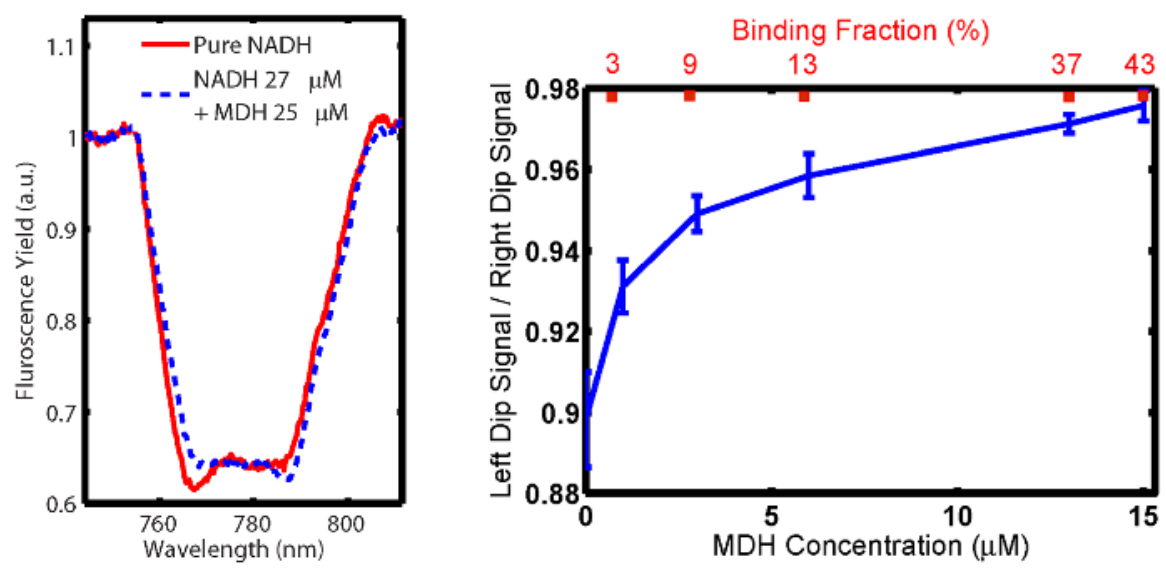

Fig. 1. Left: Normalized fluorescence as a function of $\pi$-step position for both NADH alone (solid line) and NADH with MDH (dashed line). The fluorescence from the Trizma buffer and $\mathrm{MDH}$ alone were negligible and did not affect the control. Right: Ratio of the fluorescence signal at the short-wavelength minimum to that at the long-wavelength minimum as a function of enzyme concentration. The binding fraction (as calculated based on [7]) is shown on the top of the graph. The error bars show the standard deviation of the mean for repeated measurements.

For example, as Figure 1 illustrates, we are sensitive to binding fractions as low as 3\%, with the sensitivity largely limited by the amount of signal averaging that one is able to perform. This selectivity enables one to create high-contrast fluorescent images of samples containing 
multiple fluorophores. The discrimination exploits the combination of the laser's broad bandwidth, allowing access to many possible spectral features, and finer resolution in the pulse shaper, which permits control over adjacent features. In the time domain, a structured ultrafast pulse cooperates with fast molecular dynamics to discriminate between final states.



Fig. 2. Left: Normalized fluorescence as a function of $\pi$-step position for enzyme-bound NADH at room temperature (solid line), warmed to $40^{\circ} \mathrm{C}$ (dash-dot line), and cooled back down to room temp (dash line). Right: Similar for the NADH-alone sample.

We have also investigated the usefulness of the technique for monitoring changes in binding due to modifications in the local surroundings (e.g. temperature or salinity). As shown in Figure 2 (left panel), we can follow the unbinding and rebinding of the enzyme as a function of temperature in real-time, implying that the technique could be used to monitor dynamics during environmental changes. No change in signal is seen in the NADH-alone sample (Figure 2 , right panel).

\section{Summary}

In conclusion, we have shown how pulse shaping in conjunction with multiphoton absorption can be used to distinguish between unbound and enzyme-bound biomolecules in solution. The control exploits different responses of the two forms to spectral phase shaping of the excitation light, and the results are promising for the prospect of discrimination-based microscopy. This work was supported by the National Science Foundation under Grant No. 0854922 and Dickinson College.

\section{References}

1. M. Roth, et al., Phys. Rev. Lett. 102, 253001 (2009)

2. W. Wohlleben, T. Buckup, J. L. Herek, and M. Motzkus, ChemPhysChem 6, 850 (2005)

3. K. A. Kasischke, et al., Science 305, 99 (2004)

4. Developmental Resource for Biophysical Imaging Opto-Electronics (DRBIO) at Cornell University, http://www.drbio.cornell.edu/cross sections.html

5. C. Tseng, T. C. Weinacht, A. E. Rhoades, M. Murray, and B. J. Pearson, Opt. Express 19, 24638 (2011)

6. D. Meshulach and Y. Silberberg, Phys. Rev. A 60, 1287 (1999)

7. J. J. Holbrook and R. G. Wolfe, Biochemistry 11, 2499 (1972) 